Bangladesh J. Plant Taxon. 23(1): 83-85, 2016 (June)

(C) 2016 Bangladesh Association of Plant Taxonomists

\title{
A NEW VARIETY OF SOLANUM AMERICANUM MILL. (SOLANACEAE), FROM EASTERN GHATS, INDIA
}

\author{
P. Murugan, C. Kalidass ${ }^{1}$ and P.C. Panda \\ Taxonomy and Conservation Division, Regional Plant Resource Centre, Bhubaneswar-15, India
}

Keywords: Solanum; New taxon; Eastern Ghats; India.

Solanum L., is economically and medicinally important genus and one of the largest genera of flowering plants with c. 1500 species distributed in all continents, except Antarctica (Vorontsova, et al., 2013). In India, the genus is represented by 48 species (Reema Kumari, 2004), of which 13 species are reported from Odisha (Saxena and Brahmam, 1995). During revisionary studies and exploration of the genus Solanum L. of Eastern Ghats, the authors came across some interesting specimens from the districts of Kandhamal, Ganjam and Gajapati in Odisha state, India. These specimens closely resemble Solanum americanum but differ by its height, stem, number of flowers, ovary and style characters (Table 1). Therefore, it is described here as a new variety of Solanum americanum Mill. var. odishensis var. nov.

Solanum americanum Mill. var. odishensis Kalidass \& P. Murugan, var. nov.

(Fig. 1).

Diagnosis: Solanum americanum var. odishensis is closely allied to Solanum americanum Mill., but differs from it in having prickly angular stem, inflorescence 5-8 flowered, style erect, densely villous in middle and curved at apex.

Type: INDIA, Odisha: Kandhamal District, G. Udayagiri Forest range, 14 July 2015, $20^{\circ} 07^{\prime} 204^{\prime \prime N}, 084^{\circ} 22^{\prime} 187^{\prime \prime E}, \pm 658 \mathrm{~m}$, Kalidass \& Murugan 18021 (Holotype: CAL!, Isotypes: $\mathrm{MH}$ !, RPRC!).

Erect annual herbs, 100-130 cm high. Stem 4-angular with prickles, branched, pubescentglabrescent; branches ascending. Leaves simple, alternate or sub-opposite, ovate or ovatelanceolate, $4-9 \times 3-5 \mathrm{~cm}$, cuneate or obtuse at base, margin entire to sinuate or sinuate-dentate, acute or acuminate at apex, pale beneath, glabrous or pubescent on both surfaces; lateral nerves 46 pairs; petioles $2-3 \mathrm{~cm}$ long, flat or terete. Inflorescence axillary or extra-axillary, umbellate or sub-umbellate cymes, 5-8 flowered. Flowers 3-5 mm long, creamy white; peduncle 2-3 cm long, slender, glabrous or pubescent; pedicels $8-10 \mathrm{~mm}$ long, slender, glabrous. Calyx 5-lobed, valvate, $1.5 \mathrm{~mm}$ in diameter, base united; calyx-lobes deltoid, each lobe less than $1 \mathrm{~mm}$ long, green, pubescent, margin ciliate, reflexed away from mature berries, persistent. Corolla valvate, 5lobed, 2-3 mm in diameter, star-like, base united; lobes ovate or lanceolate $2.5 \mathrm{~mm}$ long, reflexed, margin ciliate. Stamens 5, epipetalous; anthers lanceolate, $1 \mathrm{~mm}$ long, yellow, dithecus, basifixed and dehisces apically; filament c. $0.5 \mathrm{~mm}$ long, glabrous. Ovary globose, c. $0.8 \mathrm{~mm}$ long, glabrous; style less than $1.5 \mathrm{~mm}$ long, filiform, densely villous in the middle, curved at apex; stigma capitate, green. Berries globose, $5-8 \mathrm{~mm}$ in diameter black or dull black when ripe, glossy. Seeds 10-30, ovate or orbicular, 1.0-1.5 mm long, brownish-white, compressed.

Flowering and fruiting: May - September.

${ }^{1}$ Corresponding author. Email: kalidassindia@gmail.com 
Etymology: Solanum americanum var. odishensis is named after the state Odisha, one of plant diversity zones in Eastern Ghats, India.

Distribution: The new variety in distributed in the Odisha state of India as an Eastern Ghats element.

Habitat: Solanum americanum var. odishensis is found to grow in open scrub forest, usually near water courses and shady localities. It grows along with Lantana camara L., Sida acuta Burm. f., Sesamum indicum L., Solanum torvum Sw., Albizia lebbeck (L.) Benth., Neolamarckia cadamba (Roxb.) Bosser.

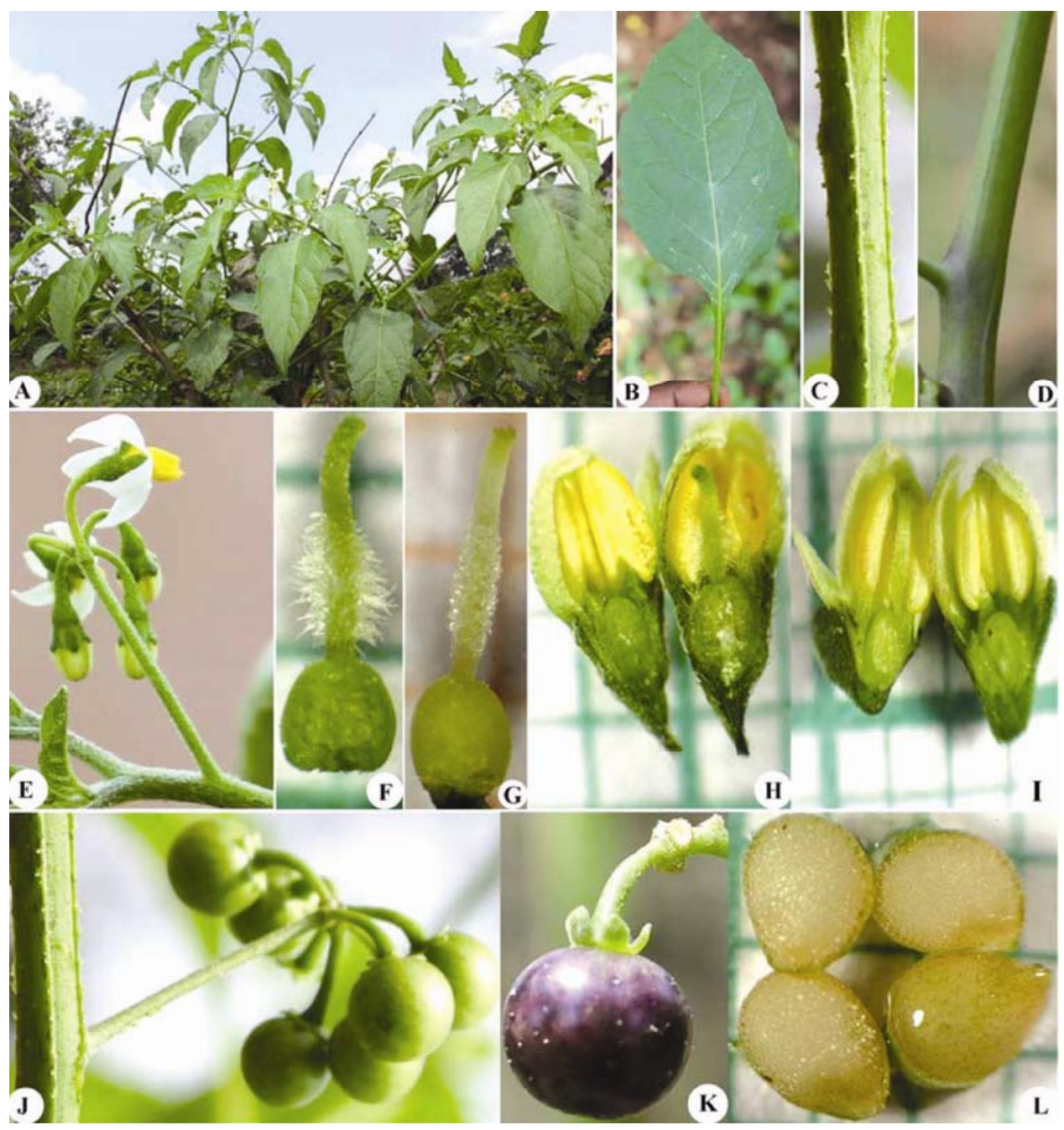

Fig. 1. Solanum americanum Mill. var. odishensis, var. nov. A. Habit; B. Leaf; C. Stem; E. Inflorescence; F. Pistil; H. L.S. of flower bud; J. Fruiting twig; K. Fruit; L. Seeds; Solanum americanum Mill. D. Stem; G. Pistil; I. L.S. of flower bud. 
Paratypes: INDIA, Odisha state, Kandhamal District, Paburia, 20 09'308"N, 084 $15^{\prime} 339^{\prime \prime} \mathrm{E}$ and Alt. \pm 681 MSL; 14.07.2015, Kalidass \& Murugan 18024 (RPRC) Kalinga Ghat, 20 $09^{\prime} 867^{\prime \prime N}$, $084^{\circ} 24^{\prime} 870^{\prime \prime} \mathrm{E}$ and Alt. \pm 737 MSL; 15.07.2015, Kalidass \& Murugan 18027 (RPRC) Ganjam District, Sikulipadara, $19^{\circ} 24^{\prime} 086^{\prime \prime} \mathrm{N}, 084^{\circ} 20^{\prime} 372$ "E and Alt. \pm 594 MSL; 19.08.2015, Kalidass \& Murugan 18045 (RPRC) Gajapati District, Parlakhemundi, Jajpur, 18 47 $630^{\prime \prime} \mathrm{N} 084^{\circ} 07^{\prime} 230^{\prime \prime E}$ and Alt. \pm 71 MSL; 21.08.2015, Kalidass \& Murugan 18067 (RPRC) Ganjam District, Taptapani, $19^{\circ} 29^{\prime} 126^{\prime \prime N}, 084^{\circ} 23^{\prime} 660^{\prime \prime E}$ and Alt. \pm 439 MSL; 22.08.2015, Kalidass \& Murugan 18087 (RPRC).

Table 1. Comparison of Solanum americanum Mill. var. odishensis var. nov. and Solanum americanum Mill. var. americanum

\begin{tabular}{lll}
\hline Character state & $\begin{array}{l}\text { Solanum americanum Mill. var. } \\
\text { odishensis var. nov. }\end{array}$ & $\begin{array}{l}\text { Solanum americanum Mill. var. } \\
\text { americanum }\end{array}$ \\
\hline Plant height & $100-130 \mathrm{~cm}$ & $\leq 100 \mathrm{~cm}$ \\
Stem & Angular, prickly & Terete, non-prickly \\
Number of flowers & $5-8$ flowered & $4-6$ flowered \\
Ovary & Globose, less than 1mm long & Ovoid, more than $1 \mathrm{~mm}$ long \\
Style & $\begin{array}{l}\text { Less than } 1.5 \mathrm{~mm} \mathrm{long,} \mathrm{densely} \\
\text { villous at middle }\end{array}$ & $\begin{array}{l}\text { More than } 1.5 \mathrm{~mm} \text { long, densely } \\
\text { glandular at middle }\end{array}$ \\
\hline
\end{tabular}

\section{Acknowledgements}

The authors are grateful to Shri. Shashi Paul, I.F.S., Chief Executive, Regional Plant Resource Centre, Bhubaneswar for their encouragement and facilities. Thanks are also due to Forest \& Environment Department, Government of Odisha, Bhubaneswar for financial support to this project.

\section{References}

Reema Kumari, M. 2004. A taxonomic revision of Indian Solanaceae. Ph. D. Thesis at Bharathiar University, Coimbatore, India.

Saxena, H.O. and Brahmam, M. 1995. The Flora of Orissa, Vol. 2. Orissa Forest Development Corporation Ltd., Bhubaneswar, pp. 1214-1223.

Vorontsova, M.S., Stern, S., Bohs, L. and Knapp, S. 2013. African spiny Solanum (Subgenus Leptostemonum, Solanaceae): a thorny phylogenetic tangle. Bot. J. Linn. Soc. 173: 176-193. 